\title{
Die Identität des Aribins mit dem Harman
}

\author{
Von \\ Ernst Späth \\ (Mit 1 Textfigur)
}

Aus dem I. Chemischen Laboratorium der Universität in Wien

(Vorgelegt in der Sitzung am 10. Juli 1919)

\section{Allgemeines,}

Im östlichen Brasilien wächst ein Baum von der Familie - der Rubiaceaen, Arariba rubra Mart. (Sickingia rubra Schumann), dessen Rinde von den Eingeborenen zum Rotfärben von Wolle verwendet wird. Vor fast 60 Jahren hat nun Rieth ${ }^{1}$ über Auftrag Wöhler's die Rinde dieses den Cinchonaarten verwandten Baumes auf den Gehalt von basischen Stoffen geprüft und hierbei ein gut charakterisiertes, festes, sauerstoffreies Alkaloid aufgefunden, das er Aribin nannte.

Ich berichte im folgenden über die wichtigsten Ergebnisse dieser Arbeit, soweit sie für die vorliegende Untersuchung von Interesse erscheinen: Das Aribin bildet farblose Krystalle, welche wasserfrei bei $229^{\circ}$ ohne Zersetzung schmelzen und dann wieder krystallinisch erstarren. Bei stärlserem Erhitzen verfüchtigt es sich ohne Zersetzung. Beim langsamen Eindunsten der ätherischen Lösung erhält man schmale, meist hohle Prismen mit 8 Molekülen Krystallwasser, bei raschem Abdampfen bleiben ziemlich große Rhombendodekaeder zurück. Die Zusammensetzung fand Rieth $z u \mathrm{C}_{23} \mathrm{H}_{20} \mathrm{~N}_{4}$. Das salzsaure

1 Rieth, Ann. d. Chem., 120, 247 (1861). 
Salz bildet glänzende Prismen, welche in Wasser leicht, in Salzsäure hingegen schwer löslich sind. Das Platinchloriddoppelsalz ist ein aus hellgelben Nadeln bestehender Niederschlag.

Irgendwelche Resultate, die zur Aufklärung der Konstitution des Aribins dienlich gewesen wären, wurden nicht ermittelt.

Seither ist über diesen Gegenstand keine Abhandlung. mehr erschienen.

Schon seinerzeit war mir bei der Durchsicht dieser Angaben aufgefallen, daß das Aribin trotz seines hohen Molekulargewichtes ohne Zersetzung sublimierbar sein sollte. Es schien mir daher wahrscheinlich, daß ein um das halbe Molekulargewicht liegender Wert der Bruttofomel größere Berechtigung besitzen könne. Bei Benützung der von Rieth ermittelten Analysenwerte und mit Berücksichtigung der Tatsache, daß bei der Verbrennung derartiger Stoffe des öfteren zu niedrige Kohlenstoffwerte erhalten werden, konnte man schließen, daß etwa die Verbindung $\mathrm{C}_{12} \mathrm{H}_{10} \mathrm{~N}_{2}$ der tatsächlichen Formel des Aribins nahekommen müsse.

Bei Durchsicht der Literatur fand ich einen Körper von der Zusammensetzung $\mathrm{C}_{12} \mathrm{H}_{10} \mathrm{~N}_{2}$, das Harman $^{1}$, das ganz ähnliche Eigenschaften besitzt wie das Aribin.

Das Harman ist ein wichtiger Abkömmling des Harmins, $\mathrm{C}_{13} \mathrm{H}_{12} \mathrm{ON}_{2}$, welches sich neben dem Harmalin, $\mathrm{C}_{13} \mathrm{H}_{14} \mathrm{ON}_{2}$, und dem Harmalol, $\mathrm{C}_{12} \mathrm{H}_{12} \mathrm{ON}_{2}$, in den Samen der in Südrußland vorkommenden Steppenraute (Peganmm hamala) vorfindet. Über diese Alkaloide ist eine größere Anzahl von Arbeiten erschienen, die in der Hauptsache von O. Fischer und seinen Schülern herrühren. Das Material zur Darstellung des Harmans, das Harmin, besitzt eine Methoxylgruppe und geht beim Erhitzen mit konzentrierter Salzsäure unter $A b-$ spaitung von Chlormethyl in die Phenoibase Harmol, $\mathrm{C}_{12} \mathrm{H}_{10} \mathrm{ON}$, Uber, aus der beim Erhitzen mit Chlorzinkammoniak auf $250^{\circ}$. unter Ersatz der Hydroxyl- gegen die Amidogruppe das Amidoharman, $\mathrm{C}_{12} \mathrm{H}_{11} \mathrm{~N}_{3}$, entsteht. Diese Verbindung gibt beim 
Diazotieren und Austausch des Diazoniumrestes gegen Wasserstoff das Harman $\mathrm{C}_{12} \mathrm{H}_{10} \mathrm{~N}_{2}$. Nach den Angaben von $\mathrm{O}$. Fischer bildet das Harman derbe Krystalle, die bei $230^{\circ}$ schmeizen, unter geringer Zersetzung sublimieren und in konzentrierter Schwefelsäure mit schwach blauer Fluoreszenz löslich sind. Die Salze fluoreszieren stark blau. Das Platinchloriddoppelsalz bildet feine hellgelbe Nadeln, das Goldchloriddoppelsalz orangegelbe, verfilzte Nädelchen.

Mit Ausnahme der Tatsache, daß in der mir zugänglichen Arbeit von Rieth ${ }^{1}$ keine Angabe von Fluoreszenz der Salze des Aribins sich vorfindet, sind die sonstigen Eigenschaften dieser Base und des Harmans so ähnlich, daß ein direkter Vergleich beider Stoffe aussichtsvoll erschien.

Das zur Untersuchung verwendete Harman verdanke ich -der Liebenswürdigkeit des Herrn Geheimrates Otto Fischer, der mir hiervon etwa $0.06 g$ zur Verfügung stellte. Aribinchlorhydrat erhielt ich durch freundliche Vermittlung des Herrn Prof. Josef Herzig von der Firma A. Schuchardt (Görlitz)-in einer Menge von $0.1 g$.

Wie ich im experimentellen Teil zeigen werde und im folgenden kurz anführe, ist die Übereinstimmung der Eigenschaften des Aribins und des Harmans und der daraus dargestellten Verbindungen eine so vollständige, daß man die Identität beider Basen als sicher annehmen kann.

Das aus Aribinchlorhydrat gewonnene Aribin schmolz nach einmaligem Umlösen aus Benzol recht scharf bei 237 bis $238^{\circ}$. Die Schmelzpunktsbestimmungen wurden zumeist in evakuierten Röhrchen ausgeführt. Das durch Sublimation gereinigte Harman schmolz nach kurz vorangehendem Sintern bei 235 bis $236^{\circ}$. Das gut verriebene Gemisch aus gleichen Teilen des reinen Aribins und des umsublimierten Harmans schmolz bei 236 bis $237^{\circ}$.

Herr Privatdozent Dr. Leitmeier hatte die Freundlichkeit, das Aribin und das Harman krystallographisch zu untersuchen. Er berichtete hierüber, daß beide Stoffe krystallographisch und optisch vollkommen identisch sind.

1.Rieth, 1. c. Sie Dissertation von Rieth, Götingen 1861 konnte jeh mir vorlitufig nicht ?versehaffen. 
Sowohl Aribin als auch Harman zeigen gleiche Löslichkeiten.

Die Chlorhydrate beider Basen sind in Wasser leicht, in Salzsäure schwer löslich.

Beide Stoffe zeigen in verdünt salzsaurer Lösung eine stark blaue Fluoreszenz von gleicher Farbtönung und Intensität.

Auch die Verbindungen des Aribins und des Harmans. waren in ihren Eigenschaften gleich.

Das Goldchloriddoppelsalz beider Basen bildet orangegelbe Krystalle, die sich beim Erhitzen im Vakuum bei $207^{\circ}$ bräunen und bei 211 bis $213^{\circ}$ unter Schwärzung und Aufschäumen schmelzen.

Die Platinchloriddoppelsalze der beiden Stoffe sind strohgelbe Nädelchen, die sich beim Eihitzen im Vakuum bei $255^{\circ}$ bis $260^{\circ}$ schwach bräunen und bei $280^{\circ}$ noch nicht. geschmolzen und erst braun gefürbt sind.

Die Pikrate von Aribin und Harman werden im Vakuum bei $215^{\circ}$ lichtbraun, bei etwa $240^{\circ}$ dunkelbraun und sind bei 250 bis $255^{\circ}$ infolge Zersetzung vollkommen schwarz.

Die Unterschiede in der Zusammensetzung beider Basen sind beim Vergleich der ermittelten Formeln so gering, daß damit nur eine weitere Bestätigung der Gleichheit beider Stoffe vorliegt. Für Aribin, für welches Rieth $\mathrm{C}_{23} \mathrm{H}_{20} \mathrm{~N}_{4}$ annahm, berechnet sich $\mathrm{C} 78 \cdot 37, \mathrm{H} 5 \cdot 72, \mathrm{~N} 15 \cdot 90 \%$, für Har$\operatorname{man} \mathrm{C}_{12} \mathrm{H}_{10} \mathrm{~N}_{2}$ C $78 \cdot 66, \mathrm{H} 5 \cdot 50, \mathrm{~N} 15.29 \%$.

Da die Formel des Harmans $\mathrm{C}_{12} \mathrm{H}_{10} \mathrm{~N}_{2}$ durch die gründlichen von $O$. Fischer ausgeführten Untersuchungen des Harmins völlig sichergestellt erscheint, ist man berechtigt, auch für das Aribin die Bruttoformel $\mathrm{C}_{12} \mathrm{H}_{10} \mathrm{~N}_{2}$ anzunehmen und die frühere Formel $\mathrm{C}_{23} \mathrm{H}_{20} \mathrm{~N}_{4} \mathrm{zu}$ streichen.

Es ist bemerkenswert, daß das Aribin, welches in einem Baume der Familie der Rubiaceaen vorkommt, identisch ist mit dem Harman, dessen Methoxylabkömmling sich in einer Pflanze der Familie der Rutaceaen vorfindet. Ich halte es für wahrscheinlich; daß das Harman auch die Stammsubstanz von anderen Alkaloiden ist, was aber wegen der Schwierigkeit 
der analytischen Behandlung derartig gebauter Verbindungen sich bisher unserer Kenntnis entzogen hat.

Uber die Konstitution des Harmans und damit auch des Aribins sind nach den bis jetzt gefundenen Forschungsresultaten nur Mutmaßungen möglich. Die Aufklärung der Konstitution des Harmans ist mit der des Harmins eng verknüpft und mit der Kenntnis des Aufbaues der einen Verbindung ist auch der andere bekannt.

Faßt man die Ergebnisse der Konstitutionsermittlung des. Harmins, die fast durchwegs ron Otto Fischer ${ }^{1}$ herrühren, zusammen, so kann man sagen, daß im Harman (Aribin) ein. trizyklisches Ringsystem vorliegt, in welchem ein Chinolinoder Isochinolinting mit einem Pyrrolkern derart kondensiert ist, daß der Pyridin- und der Pyrrolring in direkter Verbindung. miteinander stehen und worin ferner ein Wasserstoffatom des Pyridin- oder Pyrrolrestes durch eine Methylgruppe ersetzt ist. Eine Entscheidung unter den zahlreichen hier möglichen Formeln dürte nur durch die Synthese des Harmans oder des Apoharmins zu erbringen sein, die bereits von mehreren Seiten, - wenn auch bisher ohne Erfolg, in Angriff genommen wurde.

\section{Experimentelles.}

Das von der Firma Schuchardt übersandte Aribinchlorhydrat stellte ein gelblichweißes Krystallpulver dar, das sich in Wasser mit stark blauer Fluoreszenz löste. Durch Zusatz von Sodalösung und Ausschütteln mit Äther wurden fast. weiße Krystalle gewonnen, die bei der Schmelzpunktsbestimmung im offenen Röhrchen bei $235^{\circ}$ unter vorangehender. schwacher Bräunung schmolzen. Nach dem Umlösen aus wenig heißem Benzol erhielt ich kompakte Krystalle, die den

1 Otto Fischer und zum Teil seine Schüler, Ber. Deutsch. chem. Ges, $18,400(1885) ; 22,637(1889) ; 30,248(1897) ; 38.329(1905) ; 45,1930$. (19l2); 47, 99 (1914); Chem. Zentr., 1901, 1, 957. Hasenfratz, Chem. Zentr., 1912, I, 828, 1472; 1912, If, 269, 1290. Perkin und Robinson, Chem. Zentr., 1913, I, 115; 1914, I, 395.

20 . Fischer, Ber. Deutsch. chem. Ges., 22, 643 (1889) Perkin und Robinson, Joum. Chem. Soc., London, 103, 1973 (1913); Chem. Zentr.,$1914, \mathrm{I}, 395$. 
Schnelzpunkt 236 bis $237^{\circ}$ besaßen. Rieth ${ }^{1}$ fand den Schmelzpunlst des Aribins $z \mathrm{u} 229^{\circ}$.

Im Verlauf der Untersuchung wurde die Beobachtung gemacht, daß es vorteilhafter ist, die Schmelzpunktsbestimmung im Vakuum vorzunehmen, da hierbei die freien Basen Aribin and Harman vollkommen farblos bleiben und der Mischschmelzpunkt schärfer beobachtet werden konnte. Hierzu wurde ein Glasrohr von etwa $6 \mathrm{~mm}$ Durchmesser auf der einen Seite zu einer Kapillare ausgezogen und das Ende abgeschmolzen. Dann wurde die Substanz an der Stelle $a$ des Röhrchens eingefüllt, bei $b$ zu einer dickwandigen Kapillare ausgezogen, bei $c$ evakuiert und schließlich bei $b$ abgeschmolzen. Das so vorbereitete Röhrchen wurde in normaler Weise zur Schmelzpunktsbestimmung verwendet.

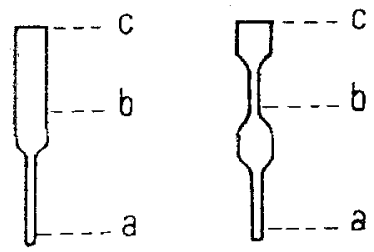

Fig. 1.

Das von Geheimrat, O. Fischer übersandte Harman war ein fast weißes Krystallpulver und schmolz im offenen Rohrchen unter vorausgehendem Sintern bei 226 bis $229^{\circ}$. Bei der Schmelzpunktsbestimmung im Vakuum, wo infolge des Farblosbleibens der Substanz eine genauere Beobachtung möglich war, sinterte das Präparat bereits ein wenig bei $212^{\circ}$, deutlich bei $220^{\circ}$ und schmolz unscharf zwischen 226 bis $230^{\circ}$. Das Produkt war also noch nicht völlig rein, da unter diesen Bedingungen das Schmelzen sicherlich ohne Zersetzung erfolgt. Darnach war entweder eine teilweise Zersetzung durch das Liegen des Stoffes eingetreten oder noch cine geringe Verunreinigung von der mit großem Geschick diirchgeführten Darstellung vorhanden. Eine teilweise Reingung konnte ich bereits im Schmelzpunktsiöhrchen erzielen; indem ich dasselbe bei $a$ vorsichtig erhitzte und dadurch einen Teil der Substanz an eine obere Stelle der Kapillare sublimicrte. Bei der neuerlichen Bestimmung mit demselben Röhrchen erweichte das sublimierte Produkt bei $229^{\circ}$ und sctimolz bei 231 bis $233^{\circ}$.

1 Rietl, 1. c. 
Bei allen Schmelzpunktsbestimmungen des Aribins und des. Harmans konnte beobachtet werden, daß die Schmelze der Basen krystallinisch erstarte und beim neuerlichen Erhitzen bei etwa 165 bis $210^{\circ}$ unscharf schmolz. Wenn man aber die erstarte Schmelze vorsichtig sublimiert, so erhält man denselben Schmelzpunkt wie zu Anfang oder sogar infolge der durch die Sublimation erzielten Reinigung einen etwas höheren.

Eine Mischprobe von reinem Aribin und dem noch unreinen Harman erweichte im Vakuum bei $228^{\circ}$ und schmolz 232 bis $235^{\circ}$. Nach dem Umsublimieren im Röhrchen schmolz das Gemisch nach kurz vorangehendem Erweichen bei 235 bis $237^{\circ}$.

Zur Reinigung des noch unreinen Harmans wurde eine kleine Menge desselben in ein einseitig geschlossenes Röhrchen gegeben, mit der Vakuumpumpe verbunden und durch vorsichtiges Erhitzen an der Flamme etwa $7 \mathrm{~cm}$ weit fraktioniert sublimiert. Man erhielt so glänzende farblose Krystalle. Der am weitesten sublimierte Anteil wurde durch Abschneiden des Röhrchens und Abkratzen vom Rest getrennt und hiervon eine Schmelzpunktsbestimmung im Vakuum vorgenommen. Die Substanz sinterte bei $232^{\circ}$ und schmolz bei 235 bis $236^{\circ}$. Der Schmelzpunkt des vollkommen reinen Harmans dürfte, nach der Sinterung zu schließen, nocin einige Grade höher liegen, doch muste wegen der geringen zur Verfügung stehenden Menge auf eine weitere Reinigung verzichtet werden.

Der Mischschmelzpunkt von dem bei 237 bis $238^{\circ}$ schmelzenden Aribin und dem bei 235 bis $236^{\circ}$ flüssig werdenden Harman lag im Vakuum bei 236 bis $237^{\circ}$ nach kurz vorangehendem Erweichen.

Auch die anderen Eigenschaften des Aribins und des Harmans und ihrer Verbindungen bestätigen die durch den: Schmelz- und Mischschmelzpunkt beider Basen ermittelte Ifentität.

Herrn Privatdozent Dr. Leitmeier (Wien) verdanke ich die krystallographische und optische Untersuchung des Aribins und des Harmans, die vorher durch möglichst gleichmäßige Sublimation im evakuierten Röhrchen gereinigt worden waren. 
Er hatte die Freundlichkeit, hierüber folgendes $z \mathrm{u}$ berichten: "Beide Stoffe sind krystallographisch und optisch vollkommen identisch. Sie sind wahtscheinlich monoklin, optisch zweiachsig, kommen in zwei Ausbildungsformen vor, die eine mehr pyramidal, die andere säulig. Die pyramidale Form ist krystallographisch besser entwickelt, läßt Pyramidenflächen erkennen und Prismenflächen nach der $a$ und $b$-Achse beobachten, aber keine Prismen nach der $c$-Achse. Die säuligen Krystalle sind nach der $c$-Achse gestreckt, zeigen eine Prismenzone nach der $c$-Achse und löschen in den untersuchten Fällen stets schief aus. Die erkennbaren Symmetrieverhältnisse an den pyramidalen Krystallen sprechen für das monokline System, womit auch der optische Befund - optisch zweiachsig übereinstimmt. Die Doppelbrechung scheint ziemlich hoch zu sein. Die säuligen Krystalle sind terminal meist schlecht begrenzt. *

Die Löslichkeiten beider Basen in Äthylalkohol, Äthyläther und Benzol sind qualitativ beurteilt gleich. Hervorzuheben ist die Schwerlöslichkeit in kaltem Benzol, die zur Reirigung Verwendung finden kann. Die Chlorhydrate von Aribin und Harman sind in Wasser ziemlich leicht löslich, werden aber durch konzentrierte Salzsäure ausgefüllt.

Aribin und Harman besitzen in verdünnt salzsaurer Lösung eine starke blaue Fluoreszenz. Bereitet man gleich konzentrierte Lösungen beider Basen, so beobachtet man gleiche Farbennuance und Stärke der Fluoreszenz.

Auch das charakteristische Goldchloriddoppelsalz beider Basen zeigte das gleiche Verhalten.

Wenn man reines Aribin in verdünnter Salzsäure unter Erwärmen löst, abkühlt und mit Natriumgoldchlorid versetzt, so erhält man eine krystallinische Fällung, die nach dem Absaugen und Trocknen in Vakuum eine orangegelbe Krystallmasse darstellt. Das Goldchloriddoppelsalz des durch Sublimation gereinigten Harmans wurde in der gleichen Weise erhalten und hatte dasselbe Aussehen. Beide Verbindungen bräunten sich im evakuierten Röhrchen bei $207^{\circ}$ und schmolzen bei $211^{\text {bis }} 213^{\circ}$ unter Schwärzung und Blasenwerfetr. Der Mischschmelzpunkt war derselbe. Das Goldchloriddoppelsalz 
ist bereits von $\mathrm{O}$. Fischer untersucht und analysiert worden. Er teschreibt es als feine verfilzte orangefarbige Nadeln.

Das Platinchloriddoppelsalz des Aribins wurde in der Weise erhalten, daß eine Lösung von reinem Aribin in verdünnter Salzsäure mit Platinchlorid versetzt wurde. Es fielen hierbei hellgelbe Nädeichen aus, die nach dem Absaugen und Waschen mit etwas Wasser im Vakuum getrocknet wurden. Die so hergestellte hellstrohgelbe Verbindung zeigte im Vakuum folgendes Verhalten: Bis $250^{\circ}$ blieb sie hell, bei 255 bis $260^{\circ}$ trat mäßige Bräunung ein und bei $280^{\circ}$ wax sie noch nicht geschmolzen und erst braun gefärbt. Die aus sublimiertem Harman in derselben Weise gewonnene Platinverbindung hatte die gleichen Eigenschaften und zeigte gleiches Verhalten beim Erhitzen im evakuierten Röhrchen.

Das Platinchloriddoppelsalz des Aribins ist bereits von Rieth, ${ }^{1}$ das des Harmans von $O$. Fischer beschrieben und analysiert worden.

Das Pikrat des Aribins erhielt ich, als ich die äthylalkoholische Lösung des reinen Aribins mit äthylalkoholischer Pikrinsäurelösung versetzte und dann mit Wasser verdünnte. Das hierbei ausgeschiedene gelbe Produkt wurde abgesaugt, mit Wasser gewaschen und im Vakuum getrocknet. In gleicher Weise wurde vom sublimierten Harman das Pikrat hergestellt. Beide Stoffe sind gelbe krystallinische Massen, die sich beim Erhitzen im evakuierten Röhrchen bei etwa $215^{\circ}$ schwach bräunen, bei $240^{\circ}$ dunkelbraun und bei 250 bis $255^{\circ}$ vollkommen schwarz werden.

1 Rieth, 1. c.

2 O. Fischer, Chem. Zentr. 1901, I, 957. 